\title{
In silico identification, characterization and expression profile of WUSCHEL-related homeobox (WOX) gene family in Vanilla planifolia
}

\author{
Thiveyarajan Victorathisayam ${ }^{1}$, Madhvi Kanchan², Himani², Thandullu R. Suriyanarayanan ${ }^{3}$, Jaspreet \\ K. Sembi ${ }^{2 *}$ \& Thakku R. Ramkumar ${ }^{2^{*}}$ \\ ${ }^{1}$ Department of Plant Biotechnology, School of Biotechnology, Madurai Kamaraj University, Madurai 625 021, Tamil Nadu, India \\ ${ }^{2}$ Department of Botany, Panjab University, Chandigarh 160 014, India \\ ${ }^{3}$ Centre of Excellence in Life Sciences, School of Life Sciences, Bharathidasan University, Thiruchirappalli 620 024, Tamil Nadu, India \\ * Email: jaspreet.sembi@pu.ac.in, ramkumartrpapers@rediffmail.com
}

\section{ARTICLE HISTORY}

Received: 03 January 2020

Accepted: 14 March 2020

Published: 18 April 2020

\section{KEYWORDS}

Vanilla planifolia

WUSCHEL

WOX genes

Gene family characterization

Expression analysis

\section{GUEST EDITOR}

Dr. Nyuk Ling Ma

Universiti Malaysia Terengganu

\section{ABSTRACT}

Vanilla planifolia is an economically important orchid, which is being commercially exploited by the food industry for the highly valued secondary metabolite vanillin. WUSCHEL-related homeobox (WOX) gene family encodes for WUSCHEL-related homeobox (WOX) transcription factors that participate in embryogenesis, organogenesis and florigenesis and in diverse plant developmental processes as well. In the present study, we analysed V. planifolia transcriptome and identified 6 WOX (VpWOX) transcripts, that encode putative WOX (VpWOX) transcription factor proteins. Domain analysis was done which indicates the presence of helix-loop-helix-turn-helix which is identifying feature of WOX gene family proteins. We executed phylogenetic clustering for the VpWOX proteins with their counterpart from the model plant Arabidopsis thaliana (AtWOX) and other closely related orchid species, Phalaenopsis equestris (PeWOX), Dendrobium catenatum (DcWOX) and Apostasia shenzhenica (AsWOX) and established their clade specific grouping. Spatio-temporal expression profile for VpWOX genes was analysed for different plant developmental stages which shows that VpWOX13 is expressing uniformly in all the developmental stages whereas, other genes have tissue specific expression. Based on gene expression patterns, we selected four VpWOX proteins and carried out secondary and tertiary structural analysis which indicates the presence of alpha helix and beta turn in the protein structure. The present study provides basic understanding of the functioning of WOX gene family in V. planifolia and paves the path for functional characterization of selected VpWOX genes in planta and in heterologous system in future for commercial utilization.

\section{Introduction}

Industrial mass production of several orchids depends on fine-tuned controlling of somatic embryogenesis and organogenesis. WUSCHEL-related homeobox (WOX) proteins are plant-specific homeobox transcription factors encoded by WUSCHEL-related homeobox (WOX) gene family members. WUSCHEL (WUS) gene was first identified in Arabidopsis thaliana (AtWUS) and plays a critical role in meristem maintenance in shoot and floral apices (1). WUS gene with a role in promoting ectopic morphogenesis, somatic embryogenesis and organogenesis have been validated in Arabidopsis thaliana (2), Coffea canephora (3) and Gossypium hirsutum (4). Vanilla planifolia is the source orchid, producing one of the most important flavour compounds vanillin. The molecular cues in embryogenesis, organogenesis and somatic embryogenesis in the recalcitrant $V$. planifolia are poorly understood. The vanillin biosynthesis depends on phenylpropanoid pathway (5). However, WOX gene family characterization in economically important orchids such as Vanilla planifolia will help in further understanding of orchid somatic embryogenesis and eventual industrial mass production. WOX proteins are characterised with 60-66 amino acid (aa) residues long homeobox domain of helix-loop-helix-turn-helix structure and DNA-binding property $(6,7)$. Phylogenetically WOX protein family members were sub-grouped into three clades as WUS, intermediate

(C) Victorathisayam et. al. (2020). This is an open-access article distributed under the terms of the Creative Commons Attribution License, which permits unrestricted use, distribution, and reproduction in any medium, provided the original author and source are credited (https://creativecommons.org/licenses/by/4.0/).

To cite this article: Victorathisayam T, Kanchan M, Himani, Suriyanarayanan TR, Sembi JK, Ramkumar TR. In silico identification, characterization and expression profile of WUSCHEL-related homeobox (WOX) gene family in Vanilla planifolia. Plant Science Today. 2020;7(2):206-213. https://doi.org/10.14719/pst.2020.7.2.722 
and ancient, the classification represents evolutionary ancestry (8). The ancient clade is present across the green lineage from unicellular algae to angiosperms, whereas the intermediate clade emerged later and is present in pteridophytes, gymnosperms and angiosperms, while the lately emerged WUS clade is found only in angiosperms (9). $W O X$ genes plays a critical role in zygote and embryonic patterning, embryogenesis, organogenesis, florigenesis and plant development by stem cell maintenance and is involved in stress response as well $(8,10)$.

The size of WOX genes family varied across plants, the model plant $A$. thaliana carries 15 genes, tool (14). MultAlin tool (15) was employed to identify the DNA-binding helix-turn-helix-loop-helix region. MEME suite online server (16), with preset parameters (maximum number of motifs - 05, number of repetitions - any, optimum motif width $\geq 6$ and $\leq 200$ ) was used to identify the conserved motifs.

\section{Physicochemical characterization}

The Expasy - ProtParam server (17) was used to determine the putative physicochemical properties such as molecular weight, aliphatic index, instability index, pI and grand average of hydropathicity (GRAVY). Sub-cellular protein localisation was predicted using online tools CELLO v.2.5 (18) and

Table 1. Ortholog prediction for VpWOX proteins

\begin{tabular}{llllll}
\hline V. planifolia & \multicolumn{1}{c}{ P. equestris } & D. catenatum & A. shenzhenica & A. thaliana \\
\hline & Protein & & & Orthologs & \\
\hline VPTC010726 & VpWOX11.1 & PeWOX11 & DcWOX11 & AsWOX11 & AtWOX11 \\
\hline VPTC010727 & VpWOX11.2 & PeWOX11 & DcWOX11 & AsWOX11 & AtWOX11 \\
\hline VPTC026011 & VpWOX9 & PeWOX9A & DcWOX9 & AsWOX9 & AtWOX9 \\
\hline VPTC018583 & VpWOX13 & PeWOX13A & DcWOX13 & AsWOX13 & AtWOX13 \\
\hline VPTC015507 & VpWOX4 & PeWOX4 & DcWOX4 & AsWOX3A & AtWOX4 \\
\hline VPTC003788 & VpWUS & PeWUS & DcWOX7 & AsWUS & AtWUS \\
\hline
\end{tabular}

Phalaenopsis equestris carries 14 genes, while the other closely related orchid species Dendrobium catenatum and Apostasia shenzhenica carry 10 genes each $(8,11,12)$. In the present study, we analysed the $V$. planifolia transcriptome and identified six transcripts encoding WOX transcription factors. Sequence similarity analysis indicated that, two transcripts are probable isoforms. Putative VpWOX proteins were characterised for their physicochemical properties. Phylogenetic relationships for VpWOX proteins were established with WOX proteins of $A$. thaliana (AtWOX), $P$. equestris (PeWOX), D. catenatum (DcWOX) and $A$. shenzhenica (AsWOX). Spatio-temporal expression of identified $V p W O X$ genes analysis suggested their critical role in plant development. No gene duplication event was predicted within VpWOX gene family. Secondary and tertiary structural analysis for four selected VpWOX proteins each representing their respective clade were performed. The results provide insights into the functional role of $V p W O X$ genes in embryogenesis, florigenesis and other plant development processes.

\section{Materials and Methods}

\section{Identification of WOX family transcripts and WOX proteins domain analysis}

To identify $V p W O X$ transcripts, WOX protein sequences from Arabidopsis thaliana (8) were used as query sequences and tblastn was carried out against transcriptome of Vanilla planifolia in Orchidstra 2.0 database (13). The VpWOX putative protein sequences obtained using $V p W O X$ transcripts were analysed and confirmed for the presence of WUSCHEL-related homeobox domain (pfam00046) by using the online SMART server, and Expasy - Prosite
WoLF PSORT (19). Protein sequences were analysed with online tools, Signal P.4.0 (20) and TMHMM v.2.0 (21) for the presence of signal peptide sequences and transmembrane helix regions respectively.

\section{Phylogenetic analysis and ortholog prediction}

The full-length sequences of putative VpWOX proteins were pre-aligned with inbuilt MUSCLE program and further analysed with MEGA7 tool (22) to establish the phylogenetic relationships between all the VpWOX proteins. The phylogenetic tree was constructed by maximum-likelihood method by using the Jones-Taylor-Thornton (JTT) model with 1000 bootstrap value. Orthologs for VpWOX protein sequences in model plant $A$. thaliana and closely related orchid species $P$. equestris, $D$. catenatum and A. shenzhenica were identified by performing local NCBI BLASTp search, with each candidate WOX protein sequences (AtWOX, PeWOX, DcWOX and AsWOX respectively) $(8,11,12)$.

\section{Gene duplication event prediction and expression analysis}

VpWOX CDS sequences were subjected to multiple sequence alignment using the online MUSCLE tool (23), and the sequences sharing $\geq 80 \%$ identity were considered as duplicate genes (24). BLASTn search was carried out using VpWOX CDS sequences as query sequences against high throughput RNA-seq data available for different developmental stages of $V$. planifolia, aerial root (SRX648209), leaf (SRX648194), vegetative bud (SRX469302), reproductive bud (SRX469303), mix bud (SRX469304) and seeds of six week old pod (SRX634907) and ten week old pod (SRX634909) in NCBI SRA database and the hit counts were noted. The RPKM values (Reads per Kilobase per Million) were calculated using the formula $\mathrm{RPKM}=\left(\mathrm{C} \times 10^{9}\right) /(\mathrm{N} \times \mathrm{L}) . \mathrm{N}$ stands for total 


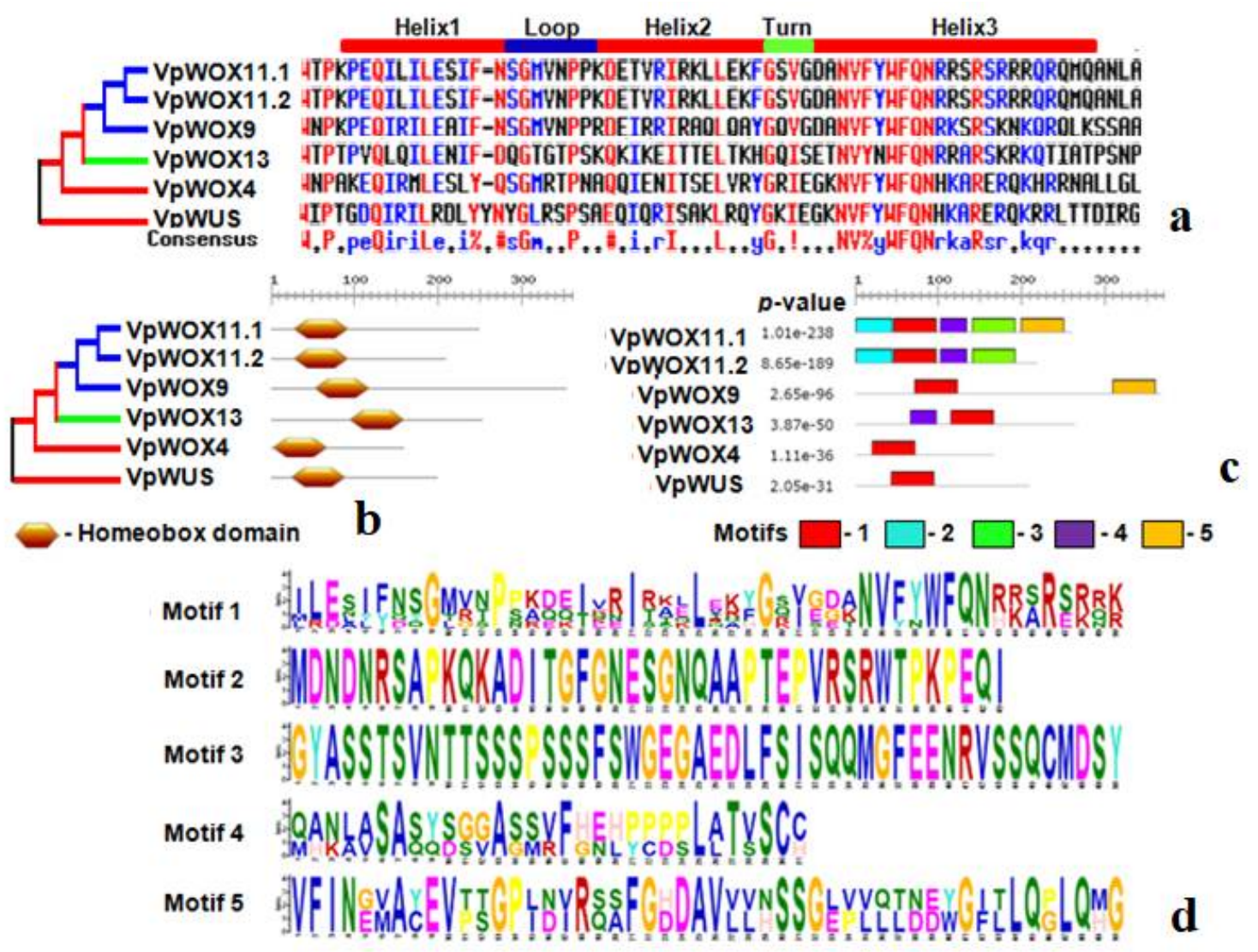

Fig. 1. Multiple sequence alignment, domain and motif analyses of VpWOX protein sequences. a Multiple sequence alignment of VpWOX sequences showing DNA-binding helix-loop-helix-turn-helix region. b VpWOX sequences showing homeobox domain. c Conserved motifs in VpWOX sequences, marked in coloured boxes. d Sequence logo of conserved motifs in VpWOX sequences, showing degree of conservation at each aa position.

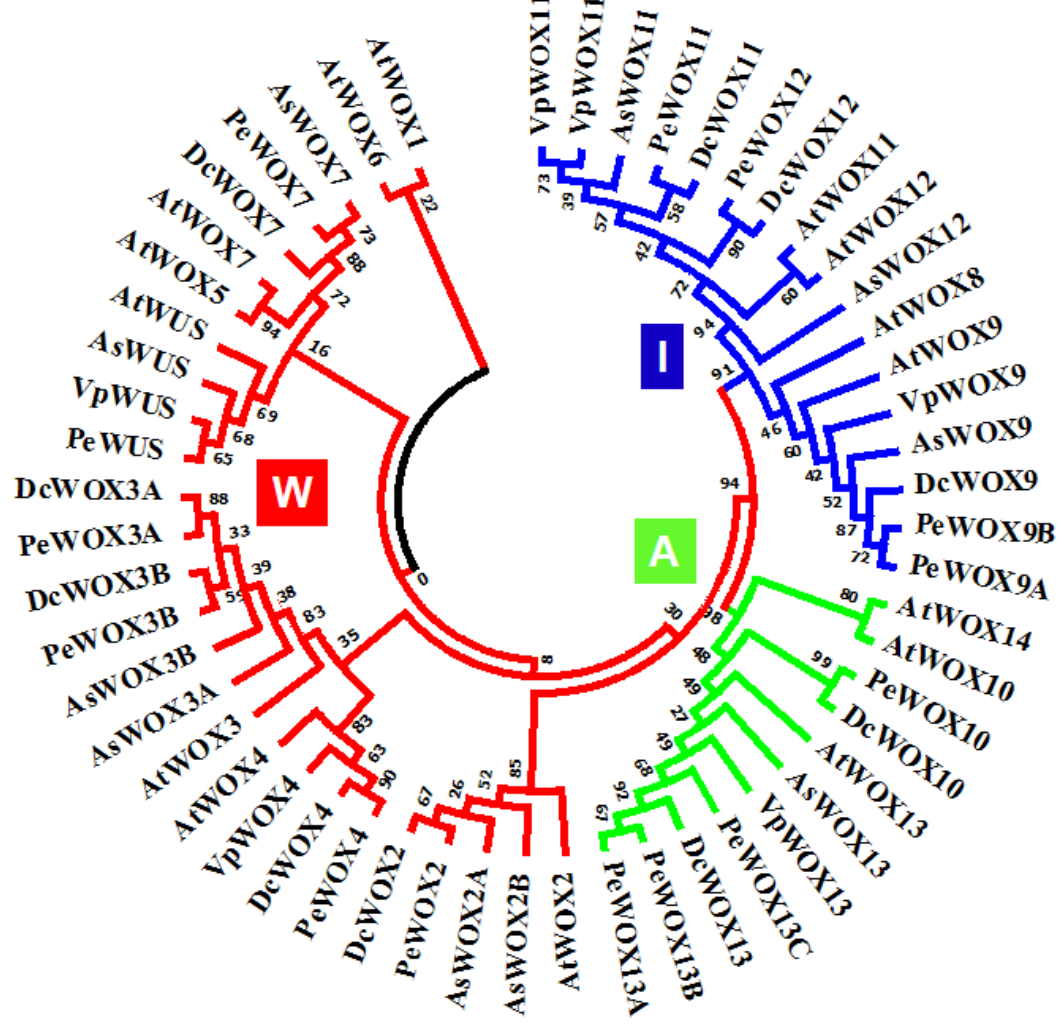

Fig. 2. Phylogenetic analysis of WOX proteins. WOX protein sequences of $V$. planifolia (VpWOX), P. equestris (PeWOX), D. catenatum (DcWOX), A. shenzhenica (AsWOX), and A. thaliana (AtWOX), are clustered phylogenetically. The ancient clade $(A)$, the intermediate clade $(I)$ and the WUS $(W)$ clade are marked respectively in green, blue and red. 
Table 2. Physico-chemical characterization of VpWOX proteins

\begin{tabular}{|c|c|c|c|c|c|c|c|c|c|}
\hline Protein & AA & MW & IP & Ins & AI & GRAVY & Loc & SP & TH \\
\hline VpWOX11.1 & 251 & 27.2 & 5.84 & 66.90 & 63.31 & -0.441 & Nucleus & No & 0 \\
\hline VpWOX11.2 & 210 & 23.0 & 9.11 & 64.69 & 53.43 & -0.693 & Nucleus & No & 0 \\
\hline VpWOX9 & 354 & 38.4 & 7.23 & 62.25 & 73.39 & -0.522 & Nucleus & No & 0 \\
\hline VpWOX13 & 254 & 28.9 & 5.49 & 57.64 & 69.45 & -0.735 & Nucleus & No & 0 \\
\hline VpWOX4 & 160 & 18.4 & 6.97 & 66.31 & 61.00 & -1.005 & Nucleus & No & 0 \\
\hline VpWUS & 201 & 22.0 & 6.83 & 58.61 & 65.17 & -0.457 & Nucleus & No & 0 \\
\hline
\end{tabular}

Sequence Id (Seq Id) from Orchidstra 2.0 database, peptide length (AA), protein molecular weight (MW) in kDa, isoelectric point (pI), instability index (Ins), aliphatic index (AI), grand average of hydropathy (GRAVY), localization (Loc), signal Peptide (SP), transmembrane domain (TMD)

Table 3. Sequence similarity index within VpWOX CDS

\begin{tabular}{|c|c|c|c|c|c|c|}
\hline Genes & VpWOX13 & VpWUS & VpWOX4 & VpWOX9 & $V p W O X 11.1$ & VpWOX11.2 \\
\hline VpWOX13 & 100 & 46.34 & 48.65 & 45.08 & 48.07 & 50.42 \\
\hline VpWUS & 46.34 & 100 & 52.75 & 49.91 & 53.19 & 53.39 \\
\hline VpWOX4 & 48.65 & 52.75 & 100 & 50.98 & 55.17 & 54.84 \\
\hline VpWOX9 & 45.08 & 49.91 & 50.98 & 100 & 62.55 & 63.93 \\
\hline VpWOX11.1 & 48.07 & 53.19 & 55.17 & 62.55 & 100 & 92.07 \\
\hline VpWOX11.2 & 50.42 & 53.39 & 54.84 & 63.93 & 92.07 & 100 \\
\hline
\end{tabular}

mapped reads in the RNA-seq experiment concerned, while the $\mathrm{L}$ stands for the base-pair length of gene and the $C$ stands for number of hits for the candidate gene (25). The heat map was generated using Hierarchical Clustering Explorer 3.5 (26).

\section{Molecular modelling}

Selected VpWOX protein sequences were analysed using SOPMA secondary structure prediction tool (27) to predict secondary structures; alpha helices, random coils, beta turns and extended strands. VpWOX protein sequences were analysed with ITasser online server (28) to predict the tertiary structure using top 10 homologous PDB templates. DNA binding site in the protein was predicted based on similar binding sites in homologous proteins. The parameters BS-scores with value of $>0.5$, TM-scores, IDEN coverage of the alignment by TM-align were considered for simulated models and binding site.

\section{Results and Discussion}

\section{Identification of VpWOX transcripts and protein domain and motif analyses}

Bioinformatics has revolutionised and put the biological research on fast-track mode. Genome and transcriptome sequencing enable researchers to identify economically important gene family members and to characterize them in silico and to further functionally characterize selected genes for commercial application. Recently $V$. planifolia transcriptome has been released by Orchidstra 2.0 database. In the present study we analysed WOX gene family with $V$. planifolia transcriptome data. $W O X$ genes are involved in plant developmental stages, particularly embryogenesis, organogenesis and florigenesis $(8,9,10)$. Extensive tBLASTn search using AtWOX proteins identified $6 \quad$ VpWOX transcripts VpWOX11.1 [VPTC010726], VpWOX11.2
[VPTC010727], VpWOX9 [VPTC026011], VpWOX13 [VPTC018583], VpWOX4 [VPTC015507] and VpWUS [VPTC003788] from $V$. planifolia transcriptome, and the naming was done depending on closest $A$. thaliana AtWOX homolog protein (Table 1). Pairwise sequence alignment analysis identified the transcripts VpWOX11.1 and VpWOX11.2 as isoforms with difference in C-terminal protein sequence. The size of WOX gene family is relatively smaller in $V$. planifolia [5] than the orchids $P$. equestris [14], $D$. catenatum [10] and $A$. shenzhenica [10] $(11,12)$.

Putative VpWOX protein sequences were generated and naming was done based on phylogenetically closest AtWOX homologs. Multiple sequence alignment indicated the occurrence of DNAbinding helix-loop-helix-turn-helix in all the six putative VpWOX protein sequences (Fig. 1a) and carried WUSCHEL-related homeobox domain (Fig. 1b). The analysis indicated that the WOX gene family was highly conserved. A total of five conserved motifs were identified in VpWOX proteins. The motif 1 represents the helix-loop-helix-turn-helix motif, present in all the VpWOX proteins which shows its highly conserved nature, which is similar to $P$. equestris and $D$. catenatum (11) (Fig. 1c, d).

\section{Phylogenetic analysis and ortholog prediction}

Phylogenetic analysis of VpWOX [6] sequences with PeWOX [14], DcWOX [10], AsWOX [10] and AtWOX [15] sequences clearly grouped the WOX proteins into three clades (Fig 2). Phylogenetical clustering of the six VpWOX proteins along with their counterparts in other plants (AtWOX, PeWOX and DcWOX) into their respective clades; VpWOX13 represents the ancient clade, VpWOX9, VpWOX11.1 and VpWOX11.2 represents the intermediate clade, while the rest two VpWUS and VpWOX4 fell in the advanced WUS clade and also indicates the conserved nature of this gene family. Orthologs for VpWOX protein sequences were identified by performing independent local BLASTp 

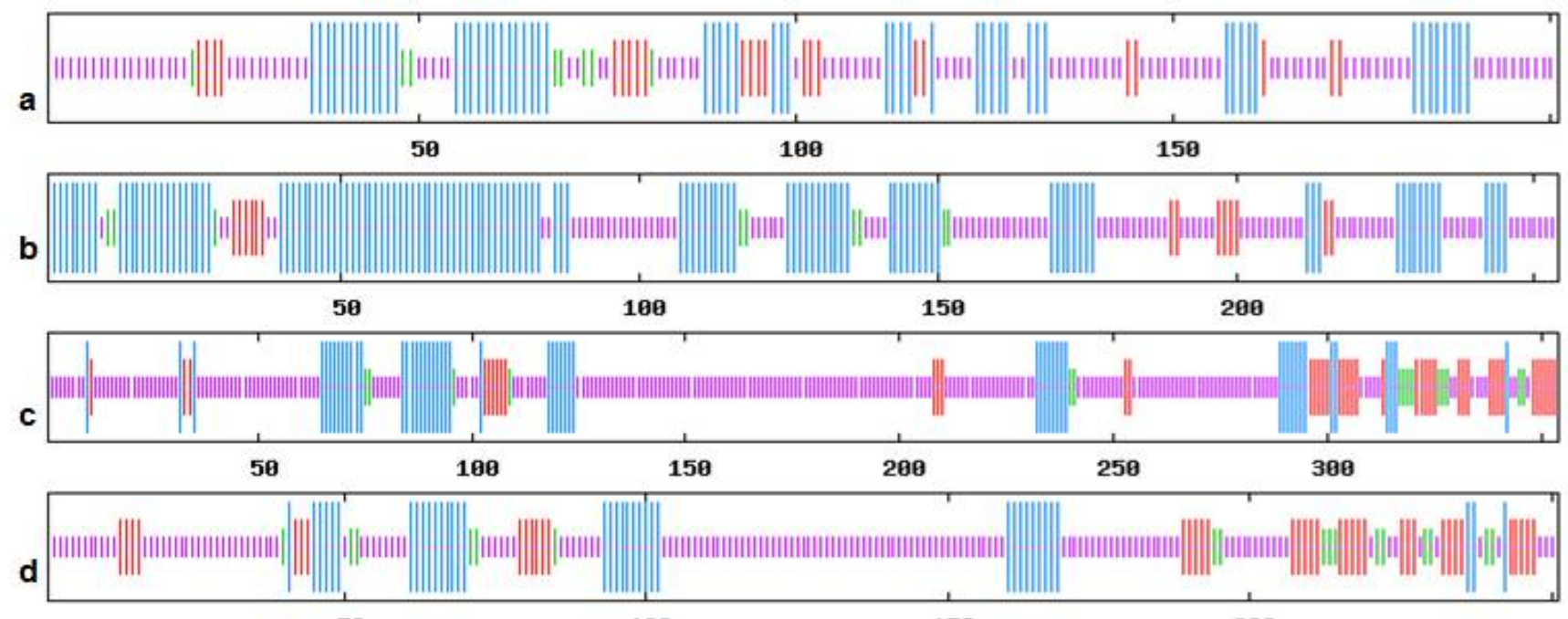

58

100

158

200
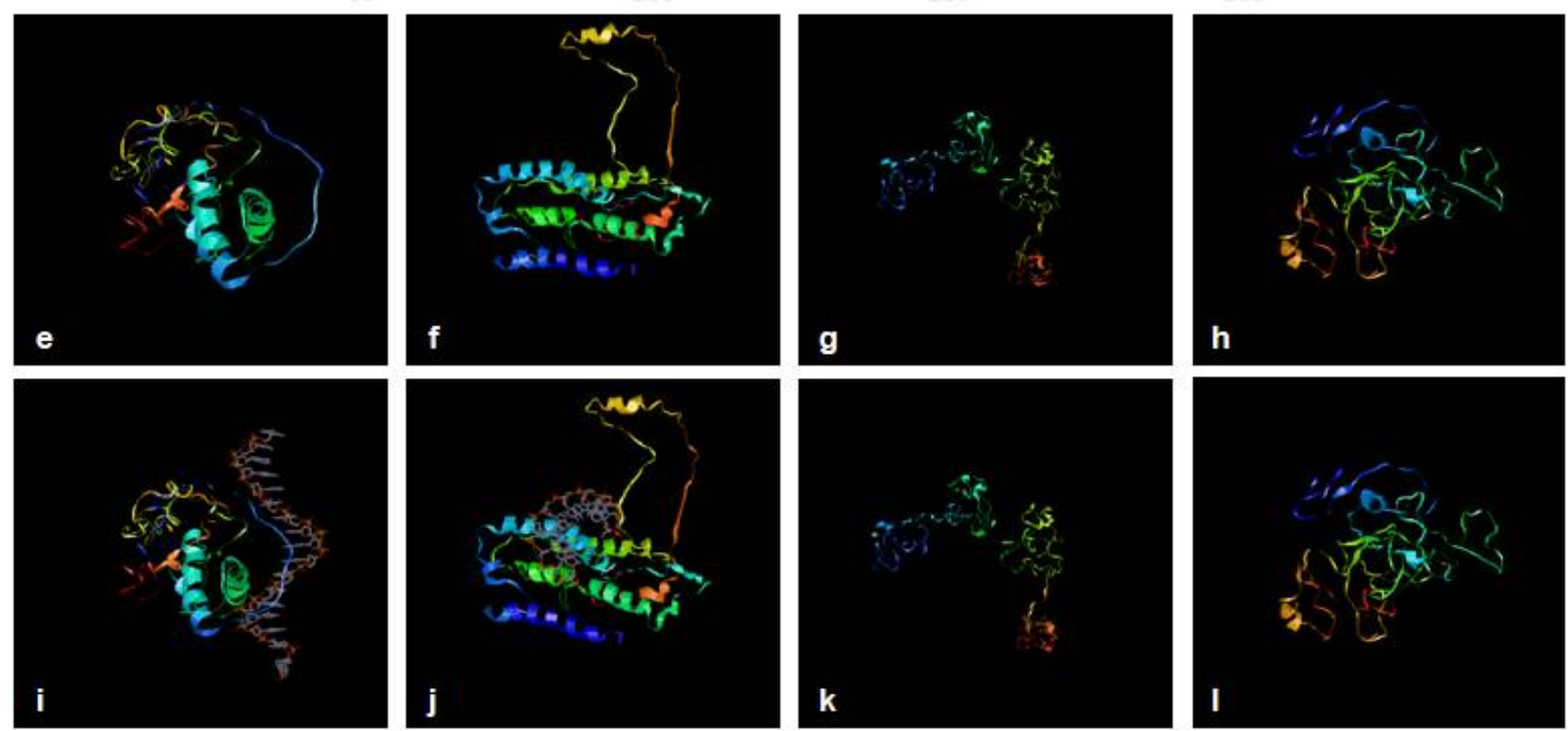

Fig. 4. Structural analysis of VpWOX proteins. Secondary structures (a, b, c, d) and simulated three-dimensional structures (e, f, g, h) with ligand-binding region (i, j, k, l) are shown for VpWOX proteins. VpWUS (a, e, i), VpWOX13 (b, f, j), VpWOX9 (c, $\mathbf{g}, \mathbf{k})$ and VpWOX11.1 $(\mathbf{d}, \mathbf{h}, \mathbf{l})$ are marked. The ligands Osmium (III) hexamine (k) and Manganese ${ }^{2+}(\mathbf{l})$ are marked with arrow.

Table 4. Secondary structure and ligand binding sites in selected VpWOX proteins

\begin{tabular}{|c|c|c|c|c|}
\hline Protein & VpWUS & VpWOX13 & VpWOX9 & VpWOX11.1 \\
\hline \multicolumn{5}{|c|}{ \% (aa) } \\
\hline$\overline{\mathrm{AH}}$ & 29.35 (59) & 49.61 (126) & $15.54(55)$ & $15.14(38)$ \\
\hline RC & $54.73(110)$ & $41.34(105)$ & $68.08(241)$ & 61.75 (155) \\
\hline ES & $11.94(24)$ & $5.51(14)$ & $12.15(43)$ & $16.33(41)$ \\
\hline$\overline{\text { BT }}$ & $3.98(8)$ & $3.54(9)$ & $4.24(15)$ & $6.77(17)$ \\
\hline$\overline{\text { LI }}$ & $\mathrm{NU}$ & $\mathrm{NU}$ & Os (III) H & $\mathrm{Mn}^{2+}$ \\
\hline$\overline{\text { BS }}$ & $6,80,83,86,87$ & $156,158,159$ & 72,75 & 9,42 \\
\hline
\end{tabular}

Alpha helix (AH), random coil (RC), extended strand (ES), beta turn (BT), ligand (LI), binding sites (BS), nucleic acid (NuASeq Id), Osmium (III) hexammine (Os (III) H), Manganese ${ }^{2+}\left(\mathrm{Mn}^{2+}\right)$

against AtWOX, PeWOX, DcWOX and AsWOX protein sequences (Table 1).

\section{Physicochemical characterization of proteins}

Physico-chemical properties for the VpWOX proteins were estimated and listed (Table 2). The average peptide length of VpWOX proteins was 238 aa, the longest VpWOX9 being 354 aa long and the shortest, the VpWOX4 was with 160 aa. The molecular weight of VpWOX proteins ranged from $18.4 \mathrm{kDa}$ to $38.4 \mathrm{kDa}$, averaging $26.3 \mathrm{kDa}$. The isoelectric point averaged 6.9 and the average aliphatic index was 64.3. The instability index ranged between 57.64 and 66.90 . All the VpWOX proteins had a negative GRAVY (grand average of hydropathy) value, a characteristic for nucleotide binding proteins. All the VpWOX proteins were predicted to be localised in the nucleus (Table 2). 


\section{Gene duplication events and spatio-temporal expression profile}

Gene duplication events are responsible for the formation of homologous genes as orthologs and paralogos. Pre-speciation gene duplication results in orthologous genes within closely related species, while post-speciation gene duplication results in paralogous gene members within the candidate species. The sequence similarity index analysis suggested that $V$. planifolia carry no gene duplication within VpWOX gene family (Table 3). Spatio-temporal expression profile of $V p W O X$ gene family members suggested, $V p W O X$ genes are involved in diverse functions. The VpWOX13 gene, of ancient clade, showed high expression in all analysed tissue except in matured seed. The expression of homologous orchid genes PeWOX13A/B/C (P. equestris), DcWOX13 (D. catenatum), AsWOX13 (A. shenzhenica) were very much similar to VpWOX13 $(11,12)$, as well as for AcoWOX13 gene in pineapple (29). AtWOX13 gene also had high expression in floral buds, inflorescences, with weak expression in leaves, fruits, in embryo development, primary and lateral root development, vegetative and fruit development and floral transition $(30,31)$. However, the PpWOX13 gene of Physcomitrella patens was reported to be involved in the reprogramming of leaf cells into stem cells (32). The observed results suggest a diverse role for VpWOX13 gene in plant development.

The VpWUS gene of WUS clade had specific expression only in mature seed and developing reproductive bud (Fig. 3). In related orchids, PeWUS gene showed pollen specific expression, AsWUS gene showed maximum expression in pollen and seed (11, 12). The $A$. thaliana AtWUS gene maintains meristem in vegetative and floral buds and regulates floral patterning $(1,33,34)$. WUS gene was shown to be involved in somatic embryo development in $C$. arabica, C. canephora and C. racemosa (35). Ectopic expression of WUS genes promoted somatic embryogenesis and organogenesis in A. thaliana (2), C. canephora (3) and Gossypium hirsutum (4). VpWOX4 gene, another member from WUS clade showed maximum expression in leaf, high expression in aerial root and mild expression in developing reproductive bud. DcWOX4 gene showed good expression in root and leaf (11). This suggests that VpWOX4 gene might have role in root development (Fig. 3).

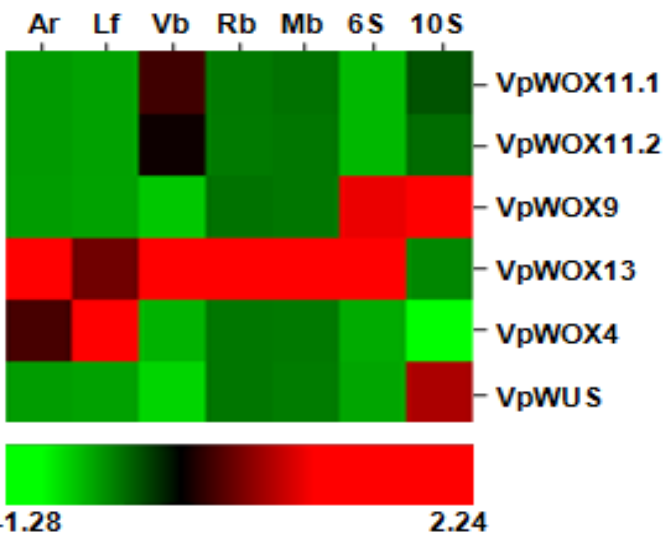

Fig. 3. Expression profile of $V p W O X$ genes. Developmental stages, Arial root (Ar), leaf (Lf), vegetative bud (Vb), reproductive bud (Rb), mixed bud (Mb) and seed stages of six week (6S) and ten weeks (10S) are marked on top.
The VpWOX9 gene of intermediate clade was highly expressive in seed of 6 week and 10 week old pod. The similar results were reported for AtWOX8 and AtWOX9 genes which are involved in zygote patterning and embryo development and act redundant to each other. Atwox8/Atwox9 double mutant showed defect in embryo development (36). This indicates VpWOX9 gene perform similar role in $V$. planifolia. The VpWOX11 gene (VpWOX11.1 and $V p W O X 11.2$ ), had moderate expression in developing vegetative bud. The expression of this gene is comparable with the AtWOX11 of A. thaliana, found to be involved in adventitious root initiation and in vitro callus initiation $(37,38)$. Similarly, in rice, the homologous OsWOX11 gene was shown to be involved in crown root development by controlling cytokinin signalling and improves drought resistance $(39,40)$. These findings from previous work suggest $V p W O X 11$ gene may have a role in vegetative growth.

\section{Homology modelling of proteins}

The homology modelling plays essential role in resolving protein structure which in-turn is necessary for understanding the mechanism of protein function. In VpWOX family four proteins VpWUS (WUS clade), VpWOX13 (ancient clade), and VpWOX9 and VpWOX11.1 (intermediate clade) were selected based on the expression profile and were analysed for structural simulation and homology modelling (Fig. 4, Table 4). The secondary structure was dominated with random coils and alpha helix regions. (Fig. 4a-d, Table 4). The VpWOX9 and VpWOX11.1 of intermediate clade had the lowest of alpha helix regions. As the WOX family members are transcription factors, WOX proteins are expected to be DNA binding. The predicted three-dimensional structures and ligand binding on the bases of their similarity with 10 homologous PDB templates of different homeodomain proteins indicates the conserved nature of these VpWOX proteins. The prediction also depicts that, the VpWUS of WUS clade and VpWOX13 of ancient clade both are nucleic acid binding transcription factors (Fig. 4e, i, f, j), whereas the VpWOX9 and VpWOX11.1 of intermediate clade are predicted to bind Osmium (III) hexamine and Manganese $^{2+}$ metal ion respectively (Fig. 4g, k, h, l). As expected VpWUS and VpWOX13 showed DNA binding. The prediction of binding of VpWOX9 to Osmium (III) hexamine is unexpected. The Osmium (III) hexamine is known to bind with nucleotides, and ribozymes (41). Similarly, VpWOX11.1 binding prediction with $\mathrm{Mn}^{2+}$ ion is also unexpected. To further confirm the metal binding properties of proteins, in-depth crystallization and in vitro studies are required. The current study is insufficient to convince the binding predictions of VpWOX9 and VpWOX11.1.

\section{Conclusion}

This present work is continuation of our previous works in model orchid Phalaenopsis equestris and closely related orchids Dendrobium catenatum and Apostasia shenzhenica. Our analysis of protein domain, motif, homology modelling and phylogenetic relationship depicts that the $V p W O X$ members are 
conserved in nature both at sequence and structural levels. The expression profile suggests that the $V p W O X$ gene family members might be playing an important role in embryogenesis, vegetative bud development and floral organ transition, as observed in other related orchids. This study will further help us in selecting candidate $V p W O X$ genes for in planta functional validation and to further generate crops with desired traits for commercial exploitation.

\section{Authors' contributions}

TRR and JKS designed the work. TV, MK, H and TRS executed the experiments. TV, MK, JKS and TRR prepared the manuscript. All authors read and approved the final manuscript.

\section{Acknowledgements}

TRR is thankful to Science and Engineering Research Board (SERB) for National-Post Doctoral Fellowship (N-PDF) (File Number: PDF/2016/003098). AV is thankful to UGC for BSR Fellowship. MK is thankful to Department of Science and Technology (DST) for INSPIRE Fellowship for Research Students. (File Number: DST/INSPIRE/03/2017/002346).

\section{Competing interests}

Authors do not have any competing interests to disclose.

\section{References}

1. Laux T, Mayer KF, Berger J, Jurgens G. The WUSCHEL gene is required for shoot and floral meristem integrity in Arabidopsis. Development. 1996;122(1):87-96. https://doi.org/10.1371/journal.pone.0038161

2. Zuo J, Niu QW, Frugis G, Chua NH. The WUSCHEL gene promotes vegetative-to-embryonic transition in Arabidopsis. The Plant Journal. 2002;30(3):349-59. https://doi.org/10.1046/j.1365-313X.2002.01289.x

3. Arroyo-Herrera A, Gonzalez AK, Moo RC, Quiroz-Figueroa FR, Loyola-Vargas VM, Rodriguez-Zapata LC, et al. Expression of WUSCHEL in Coffea canephora causes ectopic morphogenesis and increases somatic embryogenesis. Plant Cell, Tissue and Organ Culture. https://doi.org/10.1007/s11240-008-9401-1

4. Bouchabke-Coussa O, Obellianne M, Linderme D, Montes E, Maia-Grondard A, Vilaine F, et al. WUSCHEL overexpression promotes somatic embryogenesis and induces organogenesis in cotton (Gossypium hirsutum L.) tissues cultured in vitro. Plant Cell Reports. 2013;32(5):675-86. https://doi.org/10.1007/s00299-013-1402-9

5. Kundu A. Vanillin biosynthetic pathways in plants. Planta. 2017;245(6):1069-78. https://doi.org/10.1007/s00425-017-2684-x

6. Gehring WJ, Affolter M, Bürglin T. Homeodomain proteins. Annual Review of Biochemistry. 1994;63(1):487-526. https://doi.org/10.1146/annurev.bi.63.070194.002415

7. Kamiya N, Nagasaki H, Morikami A, Sato Y, Matsuoka M. Isolation and characterization of a rice WUSCHEL-type homeobox gene that is specifically expressed in the central cells of a quiescent center in the root apical meristem. The Plant Journal. 2003;35(4):429-41. https://doi.org/10.1046/j.1365313X.2003.01816.X
8. van der Graaff E, Laux T, Rensing SA. The WUS homeoboxcontaining (WOX) protein family. Genome Biology. 2009; 10(12): 248. https://doi.org/10.1186/gb-2009-10-12-248

9. Lian G, Ding Z, Wang Q, Zhang D, Xu J. Origins and evolution of WUSCHEL-related homeobox protein family in plant kingdom. The Scientific World Journal. 2014;2014 https://doi.org/10.1155/2014/534140

10. Costanzo E, Trehin C, Vandenbussche M. The role of WOX genes in flower development. Annals of Botany. 2014;114(7): 1545-53. https://doi.org/10.1093/aob/mcu123

11. Ramkumar TR, Kanchan M, Upadhyay SK, Sembi JK. Identification and characterization of WUSCHEL-related homeobox (WOX) gene family in economically important orchid species Phalaenopsis equestris and Dendrobium catenatum. Plant Gene. 2018;14:37-45. https://doi.org/10.1016/j.plgene.2018.04.004

12. Ramkumar TR, Kanchan M, Sembi JK. Genome wide characterization of WUSCHEL-related homeobox (WOX) gene family in Apostasia shenzhenica, a Primeval Orchid. Plant Science Today. https://doi.org/10.14719/pst.2020.7.2.703

13. Chao YT, Yen SH, Yeh JH, Chen WC, Shih MC. Orchidstra 2.0-a transcriptomics resource for the orchid family. Plant and Cell Physiology. 2017;58(1):e9-. https://doi.org/10.1093/pcp/pcw220

14. Sigrist CJ, De Castro E, Cerutti L, Cuche BA, Hulo N, Bridge A, Bougueleret L, Xenarios I. New and continuing developments at PROSITE. Nucleic Acids Research. 2012;41(D1):D344-7. https://doi.org/10.1093/nar/gks1067

15. Corpet F. Multiple sequence alignment with hierarchical clustering. Nucleic Acids Research. 1988;16(22):10881-90. https://doi.org/10.1093/nar/16.22.10881

16. Bailey TL, Boden M, Buske FA, Frith M, Grant CE, Clementi L, et al. MEME SUITE: tools for motif discovery and searching. Nucleic Acids Research. 2009;37(suppl_2):W202-8. https://doi.org/10.1093/nar/gkp335

17. Gasteiger E, Hoogland C, Gattiker A, Duvaud S, Wilkins MR, Appel RD, et al. Protein identification and analysis tools on the ExPASy server. The Proteomics Protocols Handbook. 2005:571607. https://doi.org/10.1385/1-59259-890-0:571

18. Yu CS, Chen YC, $\mathrm{Lu} \mathrm{CH}$, Hwang JK. Prediction of protein subcellular localization. Proteins: Structure, Function, and Bioinformatics. https://doi.org/10.1002/prot.21018

19. Horton P, Park KJ, Obayashi T, Fujita N, Harada H, AdamsCollier CJ, et al. WoLF PSORT: protein localization predictor. Nucleic Acids Research. 2007;35(suppl_2):W585-7. https://doi.org/10.1093/nar/gkm259

20. Petersen TN, Brunak S, Von Heijne G, Nielsen H. Signal P 4.0: discriminating signal peptides from transmembrane regions. Nature Methods. 2011;8(10):785 https://doi.org/10.1038/nmeth.1701

21. Krogh A, Larsson B, Von Heijne G, Sonnhammer EL. Predicting transmembrane protein topology with a hidden Markov model: application to complete genomes. Journal of Molecular Biology. 2001;305(3):567-80 https://doi.org/10.1006/jmbi.2000.4315

22. Kumar S, Stecher G, Tamura K. MEGA7: molecular evolutionary genetics analysis version 7.0 for bigger datasets. Molecular Biology and Evolution. 2016;33(7):1870 https://doi.org/10.1093/molbev/msw054

23. Edgar RC. MUSCLE: multiple sequence alignment with high accuracy and high throughput. Nucleic Acids Research. 2004 32(5):1792-97. https://doi.org/10.1093/nar/gkh340

24. Pearson WR. An introduction to sequence similarity ("homology") searching. Current Protocols in Bioinformatics. 2013;42:3-1. https://doi.org/10.1002/0471250953.bi0301s42

25. Mortazavi A, Williams BA, McCue K, Schaeffer L, Wold B. Mapping and quantifying mammalian transcriptomes by RNA$\begin{array}{lll}\text { Seq. } & \text { Nature 2008;5(7):621. }\end{array}$ https://doi.org/10.1038/nmeth.1226 
26. Seo J, Gordish-Dressman H, Hoffman EP. An interactive power analysis tool for microarray hypothesis testing and generation. Bioinformatics. https://doi.org/10.1093/bioinformatics/btk052

27. Sapay N, Guermeur Y, Deléage G. Prediction of amphipathic inplane membrane anchors in monotopic proteins using a SVM classifier. BMC bioinformatics. 2006;7(1):255. https://dx.doi.org/ $10.1186 \% 2 F 1471-2105-7-255$

28. Yang J, Yan R, Roy A, Xu D, Poisson J, Zhang Y. The I-TASSER Suite: protein structure and function prediction. Nature Methods. 2015;12(1):7. https://doi.org/10.1038/nmeth.3213

29. Rahman ZU, Azam SM, Liu Y, Yan C, Ali H, Zhao L, et al. Expression profiles of Wuschel-related homeobox gene family in pineapple (Ananas comosus L). Tropical Plant Biology. 2017; 4(10):204-15. https://doi.org/10.1007/s12042-017-9192-9

30. Deveaux Y, Toffano-Nioche C, Claisse G, Thareau V, Morin H, Laufs P, et al. Genes of the most conserved WOX clade in plants affect root and flower development in Arabidopsis. BMC Evolutionary Biology. 2008;8(1):291. https://doi.org/10.1186/1471-2148-8-291

31. Romera-Branchat M, Ripoll JJ, Yanofsky MF, Pelaz S. The WOX 13 homeobox gene promotes replum formation in the Arabidopsis thaliana fruit. The Plant Journal. 2013;73(1):37-49. https://doi.org/10.1111/tpj.12010

32. Sakakibara K, Reisewitz P, Aoyama T, Friedrich T, Ando S, Sato $\mathrm{Y}$, et al. WOX13-like genes are required for reprogramming of leaf and protoplast cells into stem cells in the moss Physcomitrella patens. Development. 2014;141(8):1660-70. https://doi.org/10.1242/dev.097444

33. Colombo L, Battaglia R, Kater MM. Arabidopsis ovule development and its evolutionary conservation. Trends in Plant Science. 2008;13(8):444-50 https://doi.org/10.1016/j.tplants.2008.04.011

34. Ikeda M, Mitsuda N, Ohme-Takagi M. Arabidopsis WUSCHEL is a bifunctional transcription factor that acts as a repressor in stem cell regulation and as an activator in floral patterning. The Plant Cell. 2009;21(11):3493-505. https://doi.org/10.1105/tpc.109.069997

35. Silva AT, Paiva LV, Andrade AC, Barduche D. Identification of expressed sequences in the coffee genome potentially associated with somatic embryogenesis. Genetics and Molecular Biology Research. 2013;12:1698-709. https://doi.org/10.4238/2013.May.21.1

36. Breuninger $\mathrm{H}$, Rikirsch $\mathrm{E}$, Hermann $\mathrm{M}$, Ueda $\mathrm{M}$, Laux $\mathrm{T}$. Differential expression of WOX genes mediates apical-basal axis formation in the Arabidopsis embryo. Developmental cell. 2008;14(6):867-76. https://doi.org/10.1016/j.devcel.2008.03.008

37. $\mathrm{Hu} \mathrm{X}, \mathrm{Xu} \mathrm{L}$. Transcription factors WOX11/12 directly activate WOX5/7 to promote root primordia initiation and organogenesis. Plant Physiology. 2016;172:2363-73. https://doi.org/10.1104/pp.16.01067

38. Jiang W, Zhou S, Zhang Q, Song H, Zhou DX, Zhao Y. Transcriptional regulatory network of WOX11 is involved in the control of crown root development, cytokinin signals and redox in rice. Journal of Experimental Botany. 2017;68:278798. https://doi.org/10.1093/jxb/erx153

39. Zhao Y, Hu Y, Dai M, Huang L, Zhou DX. The WUSCHEL-related homeobox gene WOX11 is required to activate shoot-borne crown root development in rice. The Plant Cell. 2009;21:736-48. https://doi.org/10.1105/tpc.108.061655

40. Cheng S, Zhou DX, Zhao Y. WUSCHEL-related homeobox gene WOX11 increases rice drought resistance by controlling root hair formation and root system development. Plant Signaling \& Behavior. 2016;11(2):e1130198 https://doi.org/10.1080/15592324.2015.1130198

41. Auffinger P, Grover N, Westhof E. Metal ion binding to RNA Metal Ions in Life Science. 2011;9(1). https://doi.org/10.1039/9781849732512-00001 